1 Universidade Federal de Mato Grosso (UFMT), Instituto de Saúde Coletiva, Departamento de Saúde Coletiva - Cuiabá (MT), Brasil. nereidemartinelli@gmail.com

2 Universidade de São Paulo (USP), Faculdade de Medicina, Departamento de Medicina Preventiva - São Paulo (SP), Brasil. analuizaviana@usp.br

3 Universidade Federal de Mato Grosso (UFMT), Instituto de Saúde Coletiva, Departamento de Saúde Coletiva - Cuiabá (MT), Brasil.

jscatena@ufmt.brr

\section{O Pacto pela Saúde e o processo de regionalização no estado de Mato Grosso}

\author{
The pact for health and the regionalization process in the \\ state Mato Grosso
}

Nereide Lúcia Martinelli1, Ana Luiza d'Ávila Viana², João Henrique Gurtler Scatena³
RESUMO A regionalização da saúde no estado de Mato Grosso iniciou-se nos anos 1990. Neste trabalho, analisou-se a atuação da Secretaria de Estado de Saúde, no período de 2006 a 2011, mediante as dimensões: institucionalidade, governança e impactos da regionalização, buscando identificar se as bases instituídas na vigência do Pacto facilitaram-na ou dificultaram-na. Foram utilizados dados secundários, documentos e também entrevistas com gestores estaduais, regionais e municipais. As normativas instituídas facilitaram, mas as estruturas regionalizadas tiveram suas funções modificadas; o processo de regionalização foi parcialmente implementado e impõe desafios aos entes federativos para avançar na sua consolidação.

PALAVRAS-CHAVE Regionalização; Política de saúde; Sistema Único de Saúde.

ABSTRACT The regionalization of health in the state of Mato Grosso started in the 1990s. In this work, it was analyzed the performance of the State Secretary of Health, in the period 2006-2011, by the dimensions: institutional, governance and impacts of regionalization, in order to identify whether the bases established in the presence of the Pact facilitated it or hindered it. Secondary data were used, documents and also interviews with state, region and municipal managers. The rules instituted facilitated, but the regionalized structures had their functions modified; the regionalization process was partially implemented and poses challenges to federal entities to advance in its consolidation.

KEYWORDS Regional health planning; Health policy; Unified Health System. 


\section{Introdução}

A regionalização da saúde, contemplada na Constituição de 1988, é uma das estratégias da descentralização, cujo êxito depende do compartilhamento de responsabilidades, de atos jurídico-administrativos e autonomia entre os entes federativos para provisão de serviços norteados pelo princípio da integralidade do Sistema Único de Saúde (SUS) (SANTOS; ANDRADE, 2011).

$\mathrm{Na}$ agenda de implementação do SUS, a regionalização ganha destaque nos anos 2000. Inicialmente, a descentralização valorizou a municipalização e redundou na ampliação de serviços, no incremento de resultados positivos e na maior participação popular, mas apresentou limites e dificuldades para a legitimação do direito integral à saúde (VIANA ET AL., 2010, FLEURY; OUVERNEY, 2012A).

No Pacto pela Saúde, o território regional é pensado na lógica de sistema (VIANA ET AL., 2008), requer uma nova dinâmica de relações intergovernamentais para avançar na descentralização, superar a fragmentação decisória e integrar os sistemas municipais de saúde sob a coordenação do gestor estadual (BRASIL, 2006). Com a sua implementação, esperava-se articulação, consenso, financiamento e compartilhamento entre entes federativos para, de forma planejada e corresponsável, formar redes entre as instituições e assegurar a regulação e a gestão do sistema de saúde na provisão de serviços regionalizados (VARGAS ET AL., 2014).

Segundo Abrucio (2002), pacto é uma parceria que se estabelece entre unidades territoriais para a gestão compartilhada, preservando os direitos dos pactuantes. A autonomia dos entes pode assegurar a institucionalidade e a unicidade do SUS; estabelecer relações de equilíbrio e interdependência, o exercício da governança e a cooperação, minimizando as heterogeneidades do território. Dada a complexidade das responsabilidades assumidas com a descentralização, sem a coordenação do estado há dificuldades para os entes federativos desenvolverem suas competências no SUS (VARGAS ET AL., 2014).

O território regionalizado é um espaço de intervenção que agrega municípios com diversidade em estrutura e serviços. A singularidade do modelo federativo está na maior horizontalidade das relações, que requerem ações coordenadas. Caso contrário, podem resultar em conflitos intergovernamentais, competição, interesses divergentes e até mesmo em comportamentos predatórios entre os entes envolvidos (ABRUCIO, 2002).

Este estudo analisa o processo de regionalização no estado de Mato Grosso (MT), na vigência do Pacto pela Saúde (2006-2011), verificando se as práticas instituídas e a governança impactaram nesse processo.

\section{Método}

O estado de Mato Grosso tem área de 903.366,192 km2 e uma população de 3.033.991 habitantes (IBGE, 2010), predominantemente urbana (81,9\%). Localizado na região Centro-Oeste, é constituído por 141 municípios, $80,1 \%$ deles com menos de 20 mil habitantes. Com Índice de Desenvolvimento Humano de 0,725 , posiciona-se em $11^{\circ}$ lugar entre os estados brasileiros.

Entre as 16 regiões de saúde instituídas no estado, a da Baixada Cuiabana detém $30 \%$ da população e a maior densidade demográfica, por incluir a capital, e o segundo município mais populoso - Várzea Grande. No outro extremo, seis regiões têm baixa densidade demográfica e juntas concentram pouco mais de $15 \%$ da população do estado (SCATENA ET AL., 2014A).

Desde a década de 1980, o agronegócio e a agropecuária vêm colocando MT entre as unidades da federação com as maiores taxas de expansão econômica. Em 2010, seu Produto Interno Bruto (PIB) 
per capita foi $\mathrm{R} \$ 19.636,77$ ( $14^{\mathrm{a}}$ posição nacional). Já entre as regiões do estado, o PIB variou de $R \$ 8.659,00$ a $R \$ 31.064,00$ (SCATENA ET AL., 2014A).

Excetuando-se a Atenção Básica, os estabelecimentos da rede de serviços do SUS na vigência do Pacto pela Saúde estavam concentrados no setor privado e, em sua maioria, na capital: unidades especializadas, Serviços de Apoio Diagnóstico (SADT) e serviços hospitalares. A cobertura do Programa Saúde da Família (PSF) no estado, em dezembro de 2005 , era de $56,7 \%$; em dezembro de 2010, $65,1 \%$.

A diversidade das 16 regiões de saúde é dada pelas características e pelos indicadores socioeconômicos dos municípios que as integram; há desigualdade na capacidade instalada e nos investimentos públicos e privados, sobretudo naqueles de baixo dinamismo econômico. Entre as regiões de saúde, apenas quatro têm hospitais regionais públicos (Cáceres, Rondonópolis, Sorriso e Colíder); nas demais, foram credenciados ou estabelecidos contratos com serviços privados por meio dos Consórcios Intermunicipais de Saúde (CIS).

Neste trabalho, a coleta de dados quantitativos, as entrevistas e a análise documental basearam-se em três dimensões: institucionalidade, governança e impactos da regionalização.

A institucionalidade é entendida como todas as estratégias políticas e institucionais adotadas no período; e a governança, como o conjunto de regras governamentais voltadas para a ação coletiva, que requer a divisão de poderes e o estabelecimento de relações entre atores públicos e privados, com interesses diversificados, cujas negociações podem resultar em objetivos comUnS (FLEURY; OUVERNEY, 2012B).

A institucionalidade foi analisada verificando: histórico, desenho, finalidades, estratégias políticas, planejamento, regulação e financiamento da regionalização. $\mathrm{Na}$ governança analisaram-se: estruturas de integração e gestão regional, papel do Colegiado de Gestão Regional (CGR) na regionalização e nas relações intergovernamentais. Os impactos da regionalização foram analisados considerando as mudanças institucionais observadas com a implantação do Pacto pela Saúde.

Foram utilizadas informações quantitativas do banco de dados das pesquisas 'Análise do processo de Regionalização da Saúde no Estado de Mato Grosso' e 'A regionalização da saúde no Estado de Mato Grosso: o processo de implementação e a relação público-privada na região de saúde do Médio Norte Mato-grossense', além de dados providos pelo Departamento de Informática do Sistema Único de Saúde (Datasus) e pelo Instituto Brasileiro de Geografia e Estatística (IBGE). Foram levantados documentos da Secretaria de Estado de Saúde (SES), da Comissão Intergestores Bipartite (CIB) e do governo do estado, além de outras normativas que delinearam a política de saúde no período.

As informações qualitativas foram obtidas mediante entrevistas com atores- chave da SES e de regiões de saúde, entre eles: secretário de estado da saúde; secretária executiva da CIB estadual; diretor de um Escritório Regional de Saúde (ERS); secretário de um CIS; secretária de um CGR; e secretárias de saúde de dois municípios, sendo uma delas vice-presidente regional do Conselho de Secretarias Municipais de Saúde (Cosems-MT). Para a exploração dessas informações utilizou-se a técnica de análise de conteúdo, que permitiu compreender e responder aos questionamentos sobre o processo de regionalização.

As pesquisas acima citadas foram submetidas ao comitê de ética, parecer $\mathrm{n}^{\mathrm{o}}$ 681/09 do Comitê de Ética em Pesquisa do Hospital Universitário Júlio Müller (CEP-HUJM) e parecer $\mathrm{n}^{\circ}$ 91.726/2012 do Comitê de Ética em Pesquisa da Faculdade de Medicina da Universidade de São Paulo (CEP-FMUSP). 


\section{A institucionalidade da regionalização da saúde na vigência do Pacto pela Saúde}

O início da institucionalização da regionalização da saúde em MT deu-se em meados da década de 1990, quando a SES a definiu como prioridade na política institucional da gestão estadual, no período de 1995 a 2002 (MATO GROSSO, 2000).

Tal prioridade foi expressa no documento 'Política de Saúde em Mato Grosso: diretrizes, estratégias e projetos prioritários' (MATO GROSSO, 1995), que detalha a descentralização com cooperação intergovernamental, fortalecimento da municipalização e do espaço regional. Como parte dessa política criaram-se instâncias de ação pública nos espaços regionais, entre elas as Comissões Intergestores Bipartites Regionais (CIBR), os CIS e as Câmaras de Compensação de Autorizações de Internação Hospitalar (MÜlleR NETO; LOTUFO, 2002). Os ERS tornaram-se um dos alicerces para a condução e o estabelecimento de pactos federativos regionalizados.

A SES instituiu o planejamento nos municípios e nas regiões, e, como produto dessa ação, levantou as necessidades e a capacidade de oferta por região, inserida no Plano Diretor de Regionalização (PDR) e no Plano Diretor de Investimentos (PDI) (MATO GROSSO, 2001). Na Atenção Básica foram viabilizados pactos instituídos pela SES e pelo Ministério da Saúde (MS), bem como as referências secundárias na Programação Pactuada e Integrada (PPI).

O fortalecimento da gestão regionalizada do SUS foi contemplado no Plano Plurianual (PPA) e na Lei de Diretrizes Orçamentárias (LDO) dos períodos 2004-2007 (MATO GROSSO, 2003C) e 2008-2011 (MATO GROSSO, 2010B), e, embasada neles, a SES elaborou o Plano Estadual de Saúde (PES).

Estrategicamente, a partir de 1995, o Consórcio Intermunicipal de Saúde, por indução do governo estadual, foi criado nas regiões para suprir deficiências e facilitar o acesso à assistência especializada de média complexidade ambulatorial, hospitalar e ao apoio diagnóstico. O CIS é fiscalizado pelo Tribunal de Contas do Estado e regido pelas regras de funcionamento e financiamento do estado (MATO GROSSO, 2004). A participação financeira do estado ocorre mediante convênio estabelecido entre a SES e os municípios consorciados. Já a participação do município é regulamentada por lei municipal.

O sistema estadual de regulação, criado em 1998, definiu as bases institucionais de organização do fluxo de pacientes na rede de atenção SUS. Foi criado o cargo de médico regulador do SUS, com as atribuições, entre outras, de regular a oferta de serviços de saúde e propiciar a articulação entre os diversos níveis assistenciais do sistema estadual e regional de saúde (MATO GROSSO, 2003B). Foram estabelecidos convênios com hospitais municipais para se tornarem referência regional, credenciados serviços de média e alta complexidade; e instalados leitos de Unidade de Terapia Intensiva (UTI) nas sedes de regiões, mediante convênios com unidades hospitalares próprias, municipais ou privadas.

A SES instituiu incentivos financeiros para municípios, acessados mediante o cumprimento de critérios estabelecidos nas suas portarias. Em 2003, passou a transferir recursos da receita própria, do Fundo Estadual de Saúde para os Fundos Municipais de Saúde (MATO GROSSO, 2003A).

Quando o Pacto pela Saúde foi instituído pelo governo federal em 2006, a SES elaborou o Projeto de Fortalecimento e Qualificação da Gestão Regionalizada e Solidária do SUS em Mato Grosso (FERnANDES, 2014), que contemplou a regionalização como eixo central de condução, governança e compromisso compartilhado entre os gestores, cabendo à SES a macrofunção de coordenar, articular e regular o sistema de saúde. 
Como parte desse projeto, criou-se um grupo técnico, que, em parceria com o Conselho de Secretarias Municipais de Saúde (Cosems), realizou encontros macrorregionais nas regiões de saúde, com o objetivo de ampliar e qualificar o debate da regionalização, e discutir as diretrizes e as novas responsabilidades (MATO GROSSO, 2006). O Cosems criou o projeto Rede SUS em Mato Grosso, realizou oficinas de planejamento e orçamento, e subsidiou os gestores na elaboração do Termo de Compromisso de Gestão (TCG) (RIBEIRO ET AL., 2009).

O projeto de implantação do Pacto em MT representava a possibilidade de impulsionar a regionalização, fortalecer as relações intergovernamentais e ampliar a capacidade de gestão das regiões e dos municípios para oferecer, com equidade, serviços de atenção integral. Frente às particularidades dos municípios e das regiões, implicou na redefinição de tarefas e no compartilhamento de responsabilidades expressos no TCG. O processo de pactuação entre entes federativos "envolve jogos de cooperação e competição, acordos, vetos e decisões conjuntas entre governos que possuem interesses e projetos frequentemente divergentes na disputa política" (VIANA; LIMA, 2011, P. 15).

A estruturação da rede de atenção à saúde de forma regionalizada, a articulação da SES com as macrorregiões e com os hospitais regionais, e o fortalecimento do papel dos consórcios foram objetivos contemplados no PES 2008-2011. Esse plano contemplou o fortalecimento da regionalização solidária e cooperativa, do CGR e a definição do PDR como instrumento de planejamento regional (MATO GROSSO, 2010C).

A SES, na sua macrofunção coordenadora e reguladora do sistema estadual de saúde, readequou várias vezes sua estrutura organizacional (MATO GROSSO, 2008, 2011A, 2011B, 2011C, 2013). Na estrutura vigente, coube à Gerência de Regionalização da Saúde conduzir a implementação dos instrumentos de gestão estratégicos da regionalização e apoiar o fortalecimento da gestão municipal de saúde (MATO GROSSO, 2010A).

A missão do ERS era "viabilizar o processo de descentralização da saúde, em consonância com as diretrizes do Sistema Único de Saúde" (MATO GROSSO, 2009, P. 22). Competia à essa instância: efetivar a gestão regionalizada da Política Estadual de Saúde; assessorar e monitorar os municípios no planejamento e na execução das ações; promover as pactuações regionalizadas; e coordenar os CGR. A partir de 2008, passou a contar com três gerências em sua estrutura administrativa: a de Atenção à Saúde, a de Vigilância em Saúde e a de Gestão Macrorregional (MATO GROSSO, 2008B).

A missão dessas gerências era: da Atenção à Saúde, "promover, no âmbito regional, a atenção integral à saúde [...]"; da Vigilância em Saúde, "conduzir as ações de vigilância em saúde [...] em cooperação técnica com os municípios [...]”; da Gestão Macrorregional, "gerenciar as ações relativas ao complexo regulador regional [...]" (MATO GROSSO, 2010, P. 36-37). Essas gerências facilitaram a organização interna para a cooperação técnica nos municípios. No entanto, os ERS, por falta de apoio institucional e autonomia financeira, foram deixando de realizar as atividades de cooperação e seu poder de ação ficou limitado frente às responsabilidades definidas na condução da política de saúde regionalizada. Para o gestor, o ERS "continua sempre meio que... esperando alguém dar uma ordem, não tem iniciativa... ele não está muito ativo nesta questão da regionalização" (GM).

Em 2013, essas gerências foram excluídas, mantendo-se apenas uma Gerência Técnica por ERS (MATO GROsSO, 2013). Ao extinguir a gerência de Gestão Macrorregional, alterou-se o funcionamento do Complexo Regulador Regional e das Centrais Municipais de Regulação. Em algumas regiões, a regulação da urgência e emergência foi recentralizada, ficando sob a responsabilidade da Coordenadoria de Regulação, ligada à Superintendência de 
Regulação, Controle e Avaliação da SES, em nível central.

O Plano Diretor de Regionalização (PDR) vigente para o período de 2006 a 2011 foi atualizado e homologado em 2005 (SES, 2005), com base no de 2001. O Plano Diretor de Investimentos (PDI) também foi atualizado em 2005, mas não foram encontrados registros da sua homologação na CIB/MT. Um gestor refere: "nós ainda trabalhamos com projetos e propostas de 12 anos atrás e o PDI não existe mais, não houve planejamento" (GM). O compartilhamento das responsabilidades entre entes federativos é facilitado quando há planejamento intergovernamental, quando as ações são coordenadas e reguladas para organizar os fluxos assistenciais (SILVA, 2013).

Os recursos financeiros liberados pelo MS para as regiões têm por base o PDR e o PDI vigentes. Desatualizados, tais instrumentos não serviram para organizar a rede de atenção da região e equilibrar a relação entre oferta e demanda. Além disso, não contribuíram para que os entes federativos inserissem no seu PPA, e em suas respectivas leis - LDO e Lei Orçamentária Anual (LOA) -, as necessidades de investimentos na região.

A PPI, coordenada pelo ERS e discutida no CGR, foi o instrumento de pactuação das referências e adequação do teto financeiro pré-estabelecido, que facilitou a implementação dos complexos regulatórios do sistema e o acesso às ações de saúde nas regiões e no estado. Na vigência do Pacto, o governo federal criou novos incentivos financeiros para municípios e regiões, entre os quais: compensação das especificidades regionais, colegiados de gestão regional e qualificação dos profissionais de saúde (formação técnica e educação permanente). A SES criou critérios de acesso a tais incentivos com base em indicadores, ressaltando-se: Índice de Desenvolvimento Humano (IDH), percentual de população rural, coeficiente de mortalidade infantil, índice de Gini e renda per capita. Tais recursos eram acessados mediante a elaboração de plano de ação, detalhando metas e prazos para execução e a aplicação exclusiva na melhoria da Atenção Primária à Saúde, com aprovação do CGR.

Dos incentivos criados pela SES, asseguraram-se recursos nos planos de saúde de 2004-2007 e 2008-2011 para: o Programa de Apoio e Implementação dos Consórcios (Pacis); a Microrregionalização - atividades do Centro de Atenção Psicossocial (Caps), da Hemorrede e da Reabilitação); o Programa de Apoio à Saúde Familiar e Comunitária (Pasf); o Programa Saúde Bucal na Saúde da Família; o Programa Diabetes Mellitus - Insumos Complementares; o Programa de controle da malária; o Programa de Apoio à Saúde Comunitária de Assentados Rurais (Pascar); o Programa de Incentivo ao Alcance de Metas da Atenção Básica (Piamab); e o Programa de Apoio à Organização Estadual de Urgência e Emergência. Os critérios de acesso definidos pela SES eram: cobertura de PSF; cobertura de saúde bucal; unidades cadastradas no Cadastro Nacional de Estabelecimento de Saúde (CNES); existência de Caps.

Tais incentivos financeiros deveriam ser transferidos aos municípios mensalmente, mas ocorreram com atrasos de até dez meses. Esta irregularidade fragilizou a gestão municipal, influenciou o processo de regionalização, dificultou a integração entre os entes federativos e resultou em ações isoladas, através das quais cada município procurou resolver o seu problema, apesar de sua baixa capacidade de receita própria. Entre 2007 e 2010, o Sistema de Informações sobre Orçamentos Públicos em Saúde (Siops) mostrou que os municípios aplicaram percentual acima do mínimo estabelecido pela Emenda Constitucional no 29 (EC29), próximos a $20 \%$. A fragilidade na estrutura financeira de um município leva à dependência das transferências da União e do Estado para 
implementação de sua política municipal (ARRETCHE, 2004).

Em MT, em sua maioria, os municípios são de pequeno porte e dependem da transferência de recursos financeiros, tanto do governo estadual como do federal. Para o conjunto dos municípios mato-grossenses, em 2008, as transferências do SUS representaram praticamente a metade dos recursos aplicados no setor (49\%); para o estado, elas representaram $32 \%$. Se contabilizadas as transferências interfederativas, o padrão de gasto em saúde por habitante, pelo governo estadual, foi considerado médio ou baixo ( $\mathrm{R} \$ 254,00$ ); já o gasto do conjunto dos municípios foi considerado elevado (R\$ 364,00) (VIANA ET AL., 2010; LEVI; SCATENA, 2011).

Na série histórica de 2002 a 2010, o estado cumpriu o percentual mínimo apenas no ano de 2010, atingindo 12,3\% (MENDONÇA, 2012). Sem a contrapartida financeira e a cooperação do estado, os gestores municipais não conseguem cumprir os compromissos compartilhados no TCG, em especial, a assistência de média e alta complexidade.

Em 2011, a SES realizou o chamamento público de instituições sem fins lucrativos para a celebração de contratos de gestão, para estruturas públicas estaduais (MATO GROSSO, 2011E, 2011F). Vários convênios de gestão foram estabelecidos com as organizações sociais para gerir: o Hospital Metropolitano do estado, com sede em Várzea Grande; os Hospitais Regionais de Cáceres, Rondonópolis, Colíder e Sorriso; e a Farmácia Cidadã, com sede em Cuiabá. Esta parceria repercutiu de forma negativa no sistema público de saúde do estado, gerou conflitos, questionamentos e liminares do Ministério Público Federal/MT. Tais liminares foram motivadas por denúncias e solicitações de ação civil pública, entre elas do Conselho Regional de Medicina de Mato Grosso (MATO GROSSO, 2011F). Esses contratos de gestão também repercutiram negativamente no processo de regionalização.

\section{A governança regionalizada na vigência do Pacto pela Saúde}

Dentro do formato descentralizado do SUS, a importância atribuída ao papel do Estado pode ser compreendida na representação do seu arcabouço político-institucional (GADELHA; MALDONADO; COSTA, 2012). O novo desenho conformou um sistema de saúde complexo, cuja gestão implica em distribuição de poder e responsabilidade compartilhada entre entes federativos.

O Pacto pela Saúde contemplou um novo ciclo da descentralização em MT. A governança, institucionalmente, ganhou espaço na região, evidenciando a necessidade de fortalecer as relações verticais e horizontais que conformam o complexo regional, com diversos contextos institucionais e atores com objetivos distintos, inseridos em um território de uso comum. No entanto, na sua vigência, ocorreram trocas sucessivas na gestão estadual, refletindo na maneira de gerir e conduzir a política neste ente.

A CIB, coordenada pelo secretário de estado da saúde, teve um papel importante na descentralização, um espaço de negociação que tem direcionado suas pactuações para fortalecer a regionalização (VIANA ET AL., 2010). As atas e resoluções das suas reuniões são divulgadas no site da SES. As reuniões pré-CIB e pré-CGR são bastante valorizadas pelo gestor municipal, têm o objetivo de debater temas da pauta e instrumentalizá-los para as deliberações na CIB. Conduzidas pelo Cosems, conferem importância a este conselho: "quem nos mantém informado é o Cosems” (GM).

Estudo realizado refere que no

biênio 2007-2008 as pautas da CIB foram influenciadas principalmente por: 1) políticas e demandas do MS; 2) políticas específicas e demandas da SES; 3) políticas específicas e demandas de instâncias regionais do estado. (VIANA ET AL., 2010, P. 13). 
Os principais temas de pauta estiveram relacionados à programação e à distribuição de recursos financeiros (100\% das reuniões); a políticas e estratégias de regionalização (80\%); à adesão ao Pacto (80\%); à educação permanente (80\%); a solicitações de credenciamento, serviços de alta e média complexidade, leitos hospitalares, cirurgias e outros $(60 \%)$.

Com o Pacto, as CIB regionais foram renomeadas a CGR, que consistem em um espaço institucional deliberativo e têm debatido a política de saúde, mas também influenciam as demandas dos governos federal e estadual. Nesse espaço, as negociações e decisões intergovernamentais fortaleceram parcialmente as relações horizontais e mostraram a importância de se construir objetivos comuns para a gestão cooperativa na região.

Coordenados pelo diretor do ERS, os CGR são conduzidos por regras contidas no regimento e mantidos com recursos financeiros provenientes do MS. Para os gestores, o CGR é um espaço que gera aprendizado, mas é referido da seguinte forma: "a gente não consegue ter temas transversais dentro da reunião de colegiado... é informe... muitas resoluções, proposições e portarias" (GM). Tais formalidades dificultam a governança e impedem que a discussão flua para os problemas reais vividos pelos gestores. Além disto, interferem na condução da política e não contribuem para a governabilidade sobre a macropolítica da região.

Estudos mostram que, nos locais onde "a implantação dos CGR foi conduzida de forma burocrática e cartorial, sem que tenha havido a necessária disposição para o debate por parte dos gestores", houve baixa ou nenhuma repercussão nas práticas institucionais vigentes (LIMA ET AL., 2012, P. 1911).

A Comissão Integração Ensino Serviço (Cies) estadual, instância de deliberação e decisão da política de educação permanente, foi criada em 2009, e as Cies regionais (14), a partir de 2008. Dos recursos transferidos pelo governo federal, parte tem sido executada pela Escola de Saúde de Mato Grosso para a formação técnica de profissionais. Outra parte é transferida para o Fundo de Saúde de um município da região, atendendo o Plano Regional de Educação Pemanente (Pareps). Os critérios de alocação dos recursos para as Cies regionais foram sendo alterados conforme a reformulação anual dos planos.

Nestes espaços ocorrem conflitos, e os problemas afluem, sobretudo, pela troca constante de seus membros e pela dificuldade de dispor de profissional capacitado pela Escola de Saúde Pública para realizar os cursos na região. Também por isto, muitas Cies regionais não têm executado todo o recurso financeiro contemplado no seu Pareps e nem todas Cies regionais estão atuando regularmente (VIANA ET AL., 2010).

Os Consórcios Intermunicipais de Saúde (CIS), importantes instâncias da governança regionalizada, na vigência do Pacto, conformavam 15 unidades em funcionamento no estado, englobando 125 municípios e cobrindo $62,5 \%$ da população (BOTTI ET AL., 2013). Em MT, à semelhança de outros casos no Brasil, os CIS estabeleceram parcerias entre entes federativos nas regiões e entre prestadores privados, e têm se consolidado como viabilizadores da oferta de serviços. Ampliaram a capacidade de governo municipal, sobretudo para os municípios de pequeno porte, que, sozinhos, não conseguiriam prover os serviços necessários para as suas demandas (NEVES; RIBEIRO, 2006).

Em 2008, a SES mudou a sua relação com essas instâncias. Com a justificativa de que o Pacto pela Saúde redefinia as responsabilidades de cada gestor e que os municípios necessitavam de apoio financeiro, cooperação técnica e operacional, o governo estadual editou e instituiu o Sistema de Transferência Voluntária de Recursos Financeiros do Fundo Estadual de Saúde aos Fundos Municipais de Saúde, e os municípios passaram a receber o incentivo do Pacis, fundo 
a fundo, e não por meio de convênio da SES com o CIS (MATO GROSSO, 2008A).

Com essa mudança na condução, a gerência dos consórcios foi retirada da estrutura organizacional básica e setorial da SES (MATO GROSSO, 2008B). Tais instâncias passaram a ser uma unidade não coordenada pela SES, gerando mudanças nas suas relações e com as estruturas regionalizadas (ERS, CGR). A relação da SES com o CIS é indireta, ocorre por meio da transferência da sua contrapartida financeira para o município consorciado, que a transfere para o CIS. Essa relação era mais próxima quando a gestão dos hospitais regionais era do estado (VIANA ET AL., 2010). Em algumas regiões, as equipes diretivas do CIS deixaram de participar das reuniões do CGR e das reuniões de condução e planejamento da SES (SCATENA ET AL., 2014B).

A constituição dos consórcios teve importância na regionalização de saúde em MT, fomentou a adequação da oferta à demanda, a resolubilidade e a racionalidade no uso dos serviços (VIANA ET AL., 2010), além de uma maior participação do gestor municipal na política regional. Mas a SES deixou de coordená-lo, e suas estratégias de condução não mais focaram a regionalização. O CIS deixou de ser o ator estratégico de fortalecimento da regionalização e, aos poucos, assumiu um papel de intermediador da compra e venda de serviços para o gestor, contribuindo para a fragmentação e a ineficiência do sistema público nas regiões e no estado.

No processo de regionalização, as responsabilidades foram compartilhadas por meio do TCG, mas não foram pautadas pelo planejamento integrado e pelas necessidades da população. A baixa capacidade instalada da rede de atenção nas regiões e a transferência irregular de recursos financeiros estaduais aos Fundos Municipais de Saúde contribuíram para tensionar a governança. O Estado não cumpriu com a sua parte na cooperação técnica; a falta de financiamento dificultou a regulação, influenciou a condução da política estadual de saúde, gerou conflitos nas relações intergestores e questionamentos quanto ao papel da SES na coordenação do sistema.

\section{Impactos da regionalização na vigência do Pacto pela Saúde}

As evidências levantadas mostram que, institucionalmente, a política de saúde adotada pela SES no período anterior ao Pacto foi favorável à regionalização. Sua implementação foi facilitada por vários fatores: o processo histórico estrutural da regionalização, a partir da década de 1990; o PES, que contempla a política de saúde regionalizada; a existência das CIB regionais desde 1995; a criação de novos incentivos; a concepção dos CIS; a manutenção e a instituição de novos convênios estabelecidos com serviços públicos e privados para serviços de média complexidade, serviço hospitalar e de UTI nas regiões; a participação do Cosems; a criação das CIES estadual e regional; e a indução da política financeira do MS, que exigiu organização regional e municipal. Apesar dessa institucionalidade, para os gestores, a atuação da SES "ficou muito a desejar" (GM).

O papel do ERS na coordenação e na condução da política regionalizada foi ampliado, mas, ao longo do processo, suas funções foram enfraquecidas em relação ao planejamento, à cooperação técnica e à regulação do sistema e da assistência à saúde na região. Suas relações com os gestores municipais distanciaram-se e alguns problemas municipais começaram a ser tratados diretamente com a SES e o MS.

Nas regiões, a baixa capacidade instalada, a compra dos serviços do setor privado com valores acima da tabela SUS e o atraso nas transferências financeiras do estado para os municípios repercutiram na governança regionalizada, geraram conflitos entre entes 
federativos municipais e fragilizaram a base financeira municipal.

Em algumas regiões, a regulação da urgência e emergência foi recentralizada, e as centrais regionais perderam parte de suas funções. As relações entre médicos reguladores - municipais e regional - ficaram distantes, o contato passou a ser diretamente com a central estadual. Somam-se a isso as dificuldades para regular as demandas frente à falta de capacidade instalada no estado e nas regiões. Ainda que instituídas, as bases do sistema de regulação e os instrumentos regulatórios não foram suficientes para inserir mudanças na gestão. À semelhança de outros estados, a regulação da assistência também é frágil em MT (LIMA ET AL., 2012).

Quanto à atenção primária, os incentivos financeiros instituídos pelo estado contribuíram para implementá-la, como também a parte da média e da alta complexidade, suprindo algumas demandas. $\mathrm{Na}$ assistência hospitalar, os convênios estabelecidos com o setor privado ampliaram a oferta de serviços e favoreceram o acesso, em especial, em regiões desprovidas de capacidade instalada pública. No entanto, essas atividades não foram suficientemente monitoradas $\mathrm{e}$ reguladas por parte do setor público e houve dificuldades para assegurar o acesso aos serviços complementares ao SUS.

As parcerias estabelecidas entre a SES e a Organização Social de Saúde (OSS) para gerir estabelecimentos públicos repercutiram de forma negativa. Os gestores municipais não tiveram acesso a essa gestão e atribuem a ela o motivo de atraso nas transferências financeiras do estado.

A rede estadual de atenção à saúde não foi ampliada para responder às necessidades das regiões. As resoluções da CIB/MT mostram que foram aprovados projetos para construir ou reformar unidades municipais de atenção primária de saúde, com recursos provenientes do MS e contrapartida municipal. Também foram alocados recursos federais e estaduais nas regiões de saúde para: ampliação e construção de hospitais regionais; centros cirúrgicos, leitos de estabilização e outros equipamentos hospitalares, aquisição de carros e ambulâncias; investimentos em infraestrutura de unidades não hospitalares e outros (VIANA ET AL., 2010). Uma ampliação muito mais ligada à rede de atenção municipal que à regional e as iniciativas para as regiões foram muito restritas frente às necessidades das redes regional e estadual.

Esses fatores associados às trocas sucessivas dos gestores da SES e da equipe de condução, bem como às mudanças na sua estrutura organizacional influenciaram a coordenação e o apoio aos CIS e aos ERS. O não cumprimento do percentual mínimo recomendado pela EC29, a não atualização do PDR e do PDI, além da falta de investimentos na rede pública de atenção à saúde nas regiões e no estado repercutiram na regionalização da saúde.

Os CIS ampliaram a oferta de serviços nas regiões e desenvolveram um importante papel na regionalização da saúde, em especial, na desconcentração dos serviços que estavam centrados na capital (VIANA ET AL., 2010). No entanto, as mudanças instituídas geraram rupturas nas relações e no seu modo de funcionamento. Os CIS não foram implementados e não conseguiram mobilizar e integrar suas atividades ao processo de regionalização. Em algumas regiões, eles já não constituem equipamento da regionalização.

A regionalização da saúde em MT foi influenciada positivamente pela sua institucionalidade pregressa ao Pacto, mas os problemas citados influenciaram a governança, contribuíram para comportamentos individualizados no interior dos espaços de deliberação colegiada e para relações de desconfiança e descrédito entre entes federativos, sobretudo o estadual. Observaramse, nos registros das reuniões da CIB/MT, relações de conflitos geradas por atraso nas transferências das contrapartidas 
financeiras, pela falta de hospitais regionais públicos, pela falta de acesso aos serviços na região e nas referências no estado e pela falta de planejamento.

A participação e a coordenação do estado na condução da política deveriam ser contínuas, posto que são determinantes para reduzir as desigualdades territoriais e sociais, facilitar o acesso, garantir a integralidade na atenção e potencializar a descentralização (BRASIL, 2006). No entanto, em MT, a SES, por não cumprir com as suas responsabilidades compartilhadas no TCG, contribuiu para gerar desgastes nas relações horizontais, interferiu no processo de regionalização e não fortaleceu as competências da gestão municipal. Sua participação não foi capaz de induzir a redução das desigualdades.

\section{Considerações finais}

Esta pesquisa pretendeu discutir o processo de regionalização da saúde no estado de Mato Grosso, na vigência do Pacto pela Saúde.

Ainda que a regionalização seja a estratégia indicada para reorganizar a rede de atenção e superar a fragmentação da descentralização, a governança na região mostra a dificuldade em estabelecer acordos entre entes federativos em um sistema de saúde descentralizado, para o nível municipal. Os incentivos criados pelo governo federal para a regionalização ainda são insuficientes frente às desigualdades políticas, econômicas, demográficas e financeiras das regiões. Em MT, essa situação agravou-se, pois o estado não cumpriu com as transferências mensais pactuadas com os municípios, não cumpriu na aplicação do mínimo recomendado pela EC29 e não coordenou a condução do sistema.

A política de saúde regionalizada instituída pela SES, à semelhança do apontado por outros estudos, deparou-se com fatores que mais dificultaram do que facilitaram sua condução. As evidências mostram que há desafios para: implementar a regionalização da saúde; fortalecer o processo de planejamento e regulação no estado, nas regiões e nos municípios; fortalecer a referência regional; e investir na capacidade instalada da rede de atenção regionalizada e na educação permanente, para gestores e equipe.

Por parte da SES, é preciso determinação política para cumprir o instituído, exercer a coordenação do processo, priorizar os interesses coletivos, tornar as regiões autônomas e conduzi-las de forma mais participativa. $\mathrm{O}$ projeto de regionalização em MT precisa ser reconstruído, considerando as demandas e as especificidades dos territórios, a relação e a interação dos atores, a infraestrutura, a participação e as influências existentes no espaço usado das regiões, para que se avance na sua consolidação.

\section{Referências}

ABRUCIO, F. L. Descentralização e coordenação federativa no Brasil: lições dos anos FHC. In: ABRUCIO, F. L.; LOUREIRO, M. R. (Org.). O Estado numa era de reformas: os anos FHC. Brasília, DF: SEGES, 2002, p. 143-245. parte 2 .

ARRETCHE, M. Federalismo e políticas sociais no Brasil: problemas de coordenação e autonomia. São Paulo em Perspectiva, São Paulo, v. 18, n. 2, p. 17-26, 2004.
BOTTI, C. S. et al. Regionalização dos serviços de saúde em Mato Grosso: um estudo de caso da implantação do Consórcio Intermunicipal de Saúde da região do Teles Pires, no período de 2000 a 2008. Epidemiol. Serv. Saúde, Brasília, DF, v. 22, n. 3, p. 491-500, jul./set. 2013.

BRASIL. Ministério da Saúde. Portaria GM/MS nº 399 de 22 de fevereiro de 2006. Divulga o Pacto pela Saúde 2006 - Consolidação do SUS e aprova as Diretrizes Operacionais do Referido Pacto. Diário Oficial [da] 
União. Brasília, DF, 22 fev. 2006. Disponível em: < https:// www.nescon.medicina.ufmg.br/biblioteca/registro/ Portaria_n _399_GM_de_22_de_fevereiro_de_2006_ Divulga_o_Pacto_pela_Saude_2006_e_aprova_suas_diretrizes_operacionais/56>. Acesso em: 16 out. 2015.

FERNANDES, N. F. F. Processo de organização e desenvolvimento de estratégias para divulgação e implantação do pacto pela saúde em Mato Grosso: relatório de experiência. In: SCATENA, J. H. G.; KEHRIG, R. T.; SPINELLI, M. A. (Org.). Regiões de Saúde: diversidade e processo de regionalização em Mato Grosso. SP: Hucitec, 2014, p. 135-167.

FLEURY, S.; OUVERNEY, A. M. Política de Saúde: uma política social. In: GIOVANELLA, L. et al. (Org.). Políticas e Sistema de Saúde no Brasil. 2. ed. Rio de Janeiro: Editora FIOCRUZ, 2012a, p. 25-58.

O sistema único de saúde brasileiro: desafios da gestão em rede. Revista Portuguesa e Brasileira de Gestão, Lisboa, v. 11, n. 2-3, p. 74-83, 2012 b.

GADELHA, C. A. G.; MALDONADO, J. S. S. V.; COSTA, L. S. O complexo produtivo da saúde e sua relação com o desenvolvimento: um olhar sobre a dinâmica da inovação em saúde. In: GIOVANELLA, L. et al. (Org.). Políticas e sistema de saúde no Brasil. 2 ed. Rio de Janeiro: Ed. FIOCRUZ, 2012, p. 209-239.

LEVI, M. L.; SCATENA, J. H. G. Evolução recente do financiamento do SUS e considerações sobre o processo de regionalização. In: VIANA, A. L. d’Á.; LIMA, L. D. (Org.). Regionalização e relações federativas na política de saúde do Brasil. Rio de Janeiro: Contra Capa, 2011, p. 81-113.

LIMA, L. D. et al. Descentralização e regionalização: dinâmica e condicionantes da implantação do Pacto pela Saúde no Brasil. Ciência e Saúde Coletiva, Rio de Janeiro, v. 17, n. 7, p. 1903-1914, 2012.

MATO GROSSO. Decreto ${ }^{\circ}$ 157, de 28 de fevereiro de 2011. Dispõe sobre a estrutura organizacional da Secretaria de Estado de Saúde - SES, a redistribuição de cargos em comissão e funções de confiança. Diário Oficial [do] Estado de Mato Grosso. Cuiabá, 2011a. Disponível em: <https://www.google.com.br/ url?sa $=\mathrm{t} \& \mathrm{rct}=\mathrm{j} \& \mathrm{q}=\&$ esrc $=\mathrm{s} \&$ source $=$ web $\& \mathrm{~cd}=1 \& \mathrm{ve}$ d=0CBwQFjAAahUKEwjhOu6L8pfJAhULGJAKH cU_BB8\&url=http\%3A\%2F\%2Fwww.saude.mt.gov.br\% 2Farquivo\%2F4426\%2Flegislacao\&usg=AFQjCNGCVC Sg_XA-4rHBXZ5hGk9Rebqcgw\&sig2=PES_b4i19YleBeC-fD6MIg>. Acesso em: 16 out. 2015.

. Decreto ${ }^{\circ} 259$, de 11 de abril de 2011. Dispõe sobre a estrutura organizacional da Secretaria de Estado de Saúde - SES, a redistribuição de cargos em comissão e funções de confiança. Diário Oficial [do] Estado de Mato Grosso. Cuiabá, 2011b. Disponível em: <http://www.jusbrasil.com.br/diarios/28232541/ pg-1-diario-oficial-do-estado-do-mato-grosso-doemt-de-11-04-2011>. Acesso em: 16 out. 2015.

. Decreto $n^{\circ} 669$, de 06 de setembro de 2011.

Dispõe sobre a estrutura organizacional da Secretaria de Estado de Saúde - SES, a redistribuição de cargos em comissão e funções de confiança. Diário Oficial [do] Estado de Mato Grosso. Cuiabá, 2011c. Disponível em: $<$ https://www.google.com.br/url?sa=t\&rct=j\&q=\&esrc= s\&source $=$ web $\& c d=1 \&$ cad $=$ rja $\&$ uact $=8 \&$ ved $=0 \mathrm{CBwQFj}$ AAahUKEwjMrur175fJAhXBiJAKHUDVAxk\&url=htt p\%3A\%2F\%2Fwww.saude.mt.gov.br\%2Farquivo\%2F47 82\%2Flegislacao\&usg=AFQjCNG8CHZJPSJqmnEw5p Vs7OBRCL7D0A\&sig2=NNLOwCT6hdnK3MrHewD2 9g>. Acesso em 16 out. 2015.

Decreto $n^{\circ} 765$, de 17 de junho de 2003. Dispõe sobre o Sistema de Transferência Voluntária de Financeiros do Fundo Estadual de Saúde aos Fundos Municipais de Saúde e dá outras providências. Diário Oficial [do] Estado de Mato Grosso. Cuiabá, 2003a. Disponível em: <http://app1.sefaz. mt.gov.br/Sistema/Legislacao/legfinan.nsf/2b2e6c5 ed54869788425671300480214/e075ef182926196904256 dce00513f38?OpenDocument>. Acesso em: 16 out. 2015.

. Decreto $\mathrm{n}^{\circ} 1.328$, de 14 de maio de 2008.

Dispõe sobre o Sistema de Transferência Voluntária de Recursos Financeiros do Fundo Estadual de Saúde aos Fundos Municipais de Saúde e dá outras. Diário Oficial [do] Estado de Mato Grosso. Cuiabá, 2008a. Disponível em: <https://www.google.com. $\mathrm{br} / \mathrm{url}$ ?sa $=\mathrm{t} \& \mathrm{rct}=\mathrm{j} \& \mathrm{q}=\& \mathrm{esrc}=\mathrm{s} \&$ source $=\mathrm{web} \& \mathrm{~cd}=1 \&$ ved=0CBwQFjAAahUKEwi2_ors8pfJAhVCEJAKH 
RUICzk\&url=http\%3A\%2F\%2Fwww.saude.mt.gov. br\%2Fupload\%2Flegislacao\%2F1328-\%5B1885-120110SES-MT\%5D.pdf\&usg=AFQjCNEEdDJzNdkZs6SRhq fskZEiUvMQ_A\&sig2=qrKyVrDi8-C8aK5miSMRFA>. Acesso em: 16 out. 2015.

Decreto ${ }^{\circ} 1.431$, de 03 de julho de 2008.

Dispõe sobre a estrutura organizacional da Secretaria de Estado de Saúde - SES, a redistribuição de cargos em comissão e funções de confiança. Diário Oficial [do] Estado de Mato Grosso. Cuiabá, 2008b. Disponível em: <http://www.jusbrasil.com.br/diarios/7374616/ pg-1-diario-oficial-do-estado-do-mato-grosso-doemt-de-28-07-2008>. Acesso em: 16 out. 2015.

Decreto $\mathrm{n}^{\circ}$ 1.832, de 06 de março de 2009. Aprova o Regimento Interno da Secretaria de Estado de Saúde - SES. Diário Oficial [do] Estado de Mato Grosso. Cuiabá, 2009. Disponível em: <http://www. jusbrasil.com.br/diarios/7309075/pg-3-diario-oficial-do-estado-do-mato-grosso-doemt-de-06-03-2009>. Acesso em: 16 out. 2015.

Decreto $n^{0} 1.855$, de 12 de julho de 2013. Dispõe sobre a estrutura organizacional da Secretaria de Estado de Saúde - SES, a redistribuição de cargos em comissão e funções de confiança. Diário Oficial [do] Estado de Mato Grosso. Cuiabá, 2013. Disponível em: http://www.jusbrasil.com.br/diarios/56592561/doemt12-07-2013-pg-2>. Acesso em: 16 out. 2015.

Decreto $\mathrm{n}^{\mathrm{0}} 2.916$, de 19 de outubro de 2010 . Aprova o Regimento Interno da Secretaria de Estado de Saúde - SES. Diário Oficial [do] Estado de Mato Grosso. Cuiabá, 2010a. Disponível em: <https://www. google.com.br/url?sa=t\&rct=j\&q=\&esrc $=$ s\&source $=w e b$ $\& c d=1 \& v e d=0$ CBwQFjAAahUKEwifmfKi8JfJAhVJipA KHfgEBDM\&url=http\%3A\%2F\%2Fwww.saude.mt.gov br\%2Farquivo\%2F3963\%2Flegislacao\&usg=AFQjCNG Ojev4ZM-YTD0BbhDfgzOXos4vIQ\&sig2=DjVqPSAhn 038SSgfaUEsUg>. Acesso em: 16 out. 2015.

Lei ${ }^{\circ}$ 7.990, de 07 de novembro de 2003. Cria cargos em comissão de Médico Supervisor e de Médico Regulador do Sistema Único de Saúde - SUS, no âmbito da Secretaria de Estado de Saúde. Diário Oficial [do] Estado de Mato Grosso. Cuiabá, 2003b. Disponível em:
<http://rouxinol.mt.gov.br/Aplicativos/SadLegislacao/ LegislacaoSad.nsf/709f9c981a9d9f468425671300482 be0/ff739de629bea82104256e9b00632125?OpenDocu ment>. Acesso em: 16 out. 2015.

Lei $\mathrm{n}^{\circ} 8.189$, de 28 de outubro de 2004. Dispõe sobre o funcionamento, em regime de co-gestão, de hospitais municipais que são referência de Consórcios Intermunicipais de Saúde, no âmbito do Sistema Único de Saúde do Estado de Mato Grosso. Diário Oficial [do] Estado. Cuiabá, 2004. Disponível em: <http://rouxinol.mt.gov.br/Aplicativos/SadLegislacao/LegislacaoSad.nsf/709f9c981a9d9f468425 671300482be0/flec98266le3elbc03256f510070015d? OpenDocument>. Acesso em: 16 out. 2015.

Poder Judiciário. Justiça Federal. Seção judiciária do Estado de Mato Grosso. $2^{\mathrm{a}}$ vara. Ação civil pública. Cuiabá, 2011d. Disponível em: <https://www. google.com.br/url?sa=t\&rct=j\&q=\&esrc=s\&source=w eb\&cd=1\&ved=0CBwQFjAAahUKEwirpsv69pfJAhVB CpAKHSIvAC8\&url=http\%3A\%2F\%2Fweb.trf3.jus.br \%2Fnoticias\%2Fuploaddir\%2Ffile\%2Fcoxim\%25201. pdf\&usg=AFQjCNGRKmKwbkCf2-oNCXry7MOS7b zQAQ\&sig2=og4 wPimi2llqy $6 \mathrm{fpZPuu} 4 \mathrm{~A}>$. Acesso em: 20 jul. 2014.

Secretaria de Estado da Saúde. Chamamento Público. Edital de seleção n ${ }^{\circ}$ 001, de 03 de março de 2011. Cuiabá, 2011e. Disponível em: <http://www. saude.mt.gov.br/licitacao-edital?id=1973 $>$. Acesso em: 20 jul. 2014.

Secretaria de Estado da Saúde. Chamamento Público. Edital de seleção n ${ }^{\circ}$ 003, de 18 de maio de 2011. Cuiabá, 2011f. Disponível em: <http://www.saude. $\mathrm{mt}$. gov.br/upload/documento/247/novo-edital-chamamento-publico-003-a2011-[247-190511-SES-MT]>. Acesso em: 20 jul. 2014

Secretaria de Estado da Saúde. Comissão Intergestora Bipartite Estadual. Resolução CIB $n^{\circ} 055$, de 15 de setembro de 2005. Dispõe sobre a atualização do Plano Diretor de Regionalização (PDR) do Estado de Mato Grosso. Cuiabá, 2005. Disponível em: <https:// www.google.com.br/url?sa=t\&rct=j\&q=\&esrc=s\&sour ce=web\&cd=2\&ved=0CCEQFjABahUKEwiv9srG7pfJ 
AhXEhpAKHWZODyo\&url=http\%3A\%2F\%2Fwww. saude.mt.gov.br\%2Farquivo\%2F7346\%2Flegislacao\& usg=AFQjCNFFF1Ym_cMUPDOP73MbqJNJWStsC Q\&sig2=GgwxvjiKaPfpL_JzWlYymw >. Acesso em: 16 out. 2015.

. Secretaria de Estado da Saúde. Plano Diretor de Regionalização da Assistência à Saúde - PDR. Cuiabá: SES, 2001, $336 \mathrm{p}$.

. Secretaria de Estado de Saúde. Política de Saúde em Mato Grosso: Diretrizes, Estratégias e Projetos Prioritários. Cuiabá: SES, 1995, 22 p.

Secretaria de Estado de Planejamento e Coordenação geral. PPA 2004 - 2007. Mato Grosso mãos à obra. Projeto de Lei agosto de 2003. Cuiabá: SEPLAN, 2003c, $265 \mathrm{p}$

Secretaria de Estado de Planejamento e Coordenação geral. PPA 2008 - 2011. Consolidado por objetivo estratégico. Cuibá: SEPLAN, 2010b, 482 p.

Secretaria de Estado de Saúde. Avaliação da política de saúde de Mato Grosso 1995-1998. Cuiabá: SES, 2000,188 p.

Secretaria de Estado da Saúde. Portaria n ${ }^{\circ} 26$, de 09 de março de 2006. Compõe e nomeia o Grupo Técnico de Condução e Implementação do Pacto Pela Saúde 2006 no Estado de Mato Grosso. Diário Oficial [do] Estado de Mato Grosso, 9 março de 2006, p. 33, Cuiabá, 2006.

Secretaria de Estado de Saúde.

Superintendência de Políticas de Saúde. Coordenadoria de Gestão da Política de Saúde. Plano Estadual de Saúde Mato Grosso - 2008/2011. Cuiabá: SES, 2010c. 133 p.

MENDONÇA, A. F. O financiamento do Sistema Único de Saúde nas regiões de saúde do Estado de Mato Grosso. 2012, 116f. Dissertação (Mestrado em Saúde Coletiva) Universidade Federal de Mato Grosso, Cuiabá, 2012.

MÜLLER NETO, J. S.; LOTUFO, M. Política e regionalização da saúde em Mato Grosso: aspectos históricos, conceituais, metodológicos e perspectivas. In:
MÜLLER NETO, J. S. (Org.). A regionalização da saúde em Mato Grosso: em busca da integralidade da atenção. Cuiabá: SES, 2002, p. 13-25.

NEVES, L. A.; RIBEIRO, J. M. Consórcios de Saúde: estudo de caso exitoso. Cad. Saúde Pública, Rio de Janeiro, v. 22, n. 10, p. 2207-2217, 2006.

RIBEIRO, M. S. et al. A rede de apoio ao SUS em Mato Grosso: uma estratégia para fortalecimento do papel do município na regionalização e implementação do pacto pela saúde. Divulgação em Saúde para Debate, Rio de Janeiro, n. 44, p. 62-73, maio 2009.

SANTOS, L.; ANDRADE, L. O. M. Redes Interfederativas de saúde: um desafio para o SUS nos seus vinte anos. Ciência \&t Saúde Coletiva, Rio de Janeiro, v. 16, n. 3, p.1671-1680, 2011.

SCATENA, J. H. G. et al. Caracterização das regiões de saúde de Mato Grosso. In: SCATENA, J. H. G.; KEHRIG, R. T.; SPINELLI, M. A. (Org.). Regiões de Saúde: diversidade e processo de regionalização em Mato Grosso. SP: Hucitec, 2014a, p. 135-167.

SCATENA, J. H. G. et al. A regionalização da saúde em Mato Grosso do ponto de vista dos atores regionais. In: SCATENA, J. H. G.; KEHRIG, R. T.; SPINELLI, M. A. (Org.). Regióes de Saúde: diversidade e processo de regionalização em Mato Grosso. SP: Hucitec, 2014b, p. 193-214.

SILVA, S. F. Desafios de sistemas universais de saúde sob controle público: federalismo e regionalização e o Sistema único de Saúde no Brasil. Divulgação em Saúde para Debate, Rio de Janeiro, n. 49, p. 104-113, out. 2013.

VARGAS, I. et al. Regional-based Integrated Healthcare Network policy in Brazil: from formulation to practice. [internet]. Health Policy and Planning; 2014. Disponivel em: <http://heapol.oxfordjournals.org/content/early/2014/06/23/heapol.czu048.full.pdf + html $>$. Acesso em: 20 fev. 2015.

VIANA, A. L. d'Á. et al. Novas perspectivas para a regionalização da saúde. São Paulo em Perspectiva, São Paulo, v. 22, n. 1, p. 92-106, 2008. 
Projeto de pesquisa: avaliação nacional das Comissões Intergestores Bipartites (CIBs): as CIBs e os modelos de indução da regionalização no SUS.

Relatório Estadual Mato Grosso, 2010, p. 1-20.

VIANA, A. L. d'Á.; LIMA, L. D. O processo de regionalização na saúde: contextos, condicionantes e papel das Comissões Intergestores Bipartites. In: VIANA, A.

L. d’Á.; LIMA, L. D. (Org.). Regionalização e relações federativas na política de saúde do Brasil. Rio de Janeiro. Contra Capa Livraria Ltda., 2011, p. 11-24.

Recebido para publicação em abril de 2015

Versão final em outubro de 2015

Conflito de interesses: inexistente

Suporte financeiro: não houve 